\title{
Coumarin and Safrole Content in Cinnamon-Flavored Food Products on the Syrian Market
}

\author{
Raw'aa Solaiman* and Joumaa Al-Zehouri
}

\author{
Department of Food Control and Analytical Chemistry, College of Pharmacy, Damascus University, \\ Damascus, Syria
}

\begin{abstract}
Some plants that are processed into foods often contain natural substances that may be hazardous to human health. One example is coumarin, which is known to cause liver and kidney damage in rats, mice and probably humans. Coumarin is found in different Cinnamomum species such as Cinnamomum cassia, Cinnamomum loureiroi, and Cinnamomum burmannii; all commonly referred to as cassia. Another hazardous substance is Safrole. Safrole is reasonably anticipated to be a human carcinogen based on sufficient evidence of carcinogenicity from studies in experimental animals; and is mainly present in cinnamon leaf oil and could be a possible contaminant in cinnamon powder. European Council's Directive on food flavourings 88/388/EEC limits safrole in foodstuffs to $1 \mathrm{ppm}$. The content of coumarin is regulated in the European Regulation (EC) No 1334/2008. In the present study, coumarin and safrole levels were analyzed in locally bought cinnamon samples and cinnamon-flavored food products using a validated HPLC method with diode array detector (DAD). Appreciable amounts of coumarin were found in bakery products with concentrations up to $39.466 \mathrm{mg} / \mathrm{kg}$ in certain kinds of cookies, whereas safrole was undetectable. Our exposure data on coumarin in bakery products show that there is still a need for a continued regulation of coumarin in foods. A toxicological re-evaluation of coumarin with the aim to derive scientifically founded maximum limits should be conducted with priority.
\end{abstract}

Keywords: Cinnamon, Coumarin, Safrole, High-performance liquid chromatography, cinnamon-flavored foods.

\section{INTRODUCTION}

Coumarin is a natural substance occurring in the essential oils of a number of plants used as flavoring ingredients in foods. Coumarin's aroma has been described as sweet, aromatic, creamy vanilla bean odor [1]. Until 1954, when the first toxicological concerns about coumarin were raised, synthetic coumarin was widely used to add flavour, e.g. to the so-called may wines (second-grade white wine flavored with woodruff) [1]. After that, the use of coumarin as a food flavoring was discontinued based on reports of hepatotoxicity prior to the existence of any carcinogenicity and mutagenicity data [2]. According to Lake's estimation, the main source of coumarin in the diet is cinnamon [2]. The cinnamon used domestically and industrially in the preparation of food originates primarily from true cinnamon and from cassia cinnamon (simply referred to as cassia). True cinnamon belongs to the species of Cinnamomum verum whereas cassia generally includes the species of Cinnamomum cassia, also known as Cinnamomum aromaticus, Cinnamomum loureiroi and Cinnamomum burmannii Blume [3]. Cassia is cheaper and generally more popular in Europe compared to true cinnamon [4]. True cinnamon and cassia taste different because of a

*Address of correspondence to this author at the Department of Food Control and Analytical Chemistry, College of Pharmacy, Damascus University, Damascus, Syria; Tel: +963988 010562; E-mail: rm_spo@hotmail.com different chemical composition. For example, cassia contains more of the aromatic compound coumarin (1benzopyran-2-one) compared to true cinnamon [5]. The European Food Safety Authority (EFSA) derived a tolerable daily intake (TDI) of $0-0.1 \mathrm{mg}$ coumarin $/ \mathrm{kg}$ body weight [6]. In 2005, a high content of coumarin in cookies initiated increased authoritarian coumarin control and debate [6-7]. This, in combination with a widespread and increased use of cassia in food products, led the European Parliament and Council to evaluate the maximum coumarin limits [8]. In 2008, the European Regulation (EC) No 1334/2008 was enacted with the following maximum limits for coumarin: 50 $\mathrm{mg} / \mathrm{kg}$ in traditional and/or seasonal bakery ware containing a reference to cinnamon in the labeling, 20 $\mathrm{mg} / \mathrm{kg}$ in breakfast cereals including muesli, $15 \mathrm{mg} / \mathrm{kg}$ in fine bakery ware, with the exception of traditional and/or seasonal bakery ware containing a reference to cinnamon in the labeling, and $5 \mathrm{mg} / \mathrm{kg}$ in desserts. Coumarin should not be added as such to food products [9].

Safrole \& sassafras oil were banned as food \& flavoring additives by the FDA on 3rd Dec 1960 [10]. The ban now includes isosafrole \& dihydrosafrole (the latter not being known in nature), \& sassafras root bark, but in practice both sassafras oil and bark are still widely available in the US, from health food stores and internet suppliers [11]. European Council's Directive on food flavourings 88/388/EEC [12], amended by 
91/71/EEC and implemented into UK national law in the Flavourings in Food Regulations 1992, limits safrole in foodstuffs to $1 \mathrm{ppm}$, except for foodstuffs containing nutmeg $(15 \mathrm{ppm})$ or alcoholic drinks $>25 \%$ volume alcohol (5ppm) and other alcoholic drinks (2ppm) [12]. Quantification of coumarin and safrole is primarily performed with chromatographic techniques such as high performance liquid chromatography (HPLC) [13], gas chromatography (GC) [14], and the more recent introduction of ultra performance liquid chromatography (UPLC) [7]. This paper describes an HPLC method and investigates coumarin and safrole content in Syrian food products with cinnamon in relation to the EU limits.

\section{MATERIALS AND METHODS}

\subsection{Chemicals and Standards}

Coumarin ( $\geq 99 \%)$, Safrole ( $\geq 97 \%$ ) and glacial acetic acid $99.7 \%$ were purchased from Sigma Aldrich (Germany). Acetonitrile (HPLC grade) and Methanol (HPLC grade) were purchased from Fisher Scientific (United Kingdom). Disposable syringe filters with a pore width of $0.2 \mu \mathrm{m}$ (Chromafil PET-20/25) were purchased from Macherey-Nagel (Germany).

\subsection{HPLC Instrumentation and Chromatographic Conditions}

The HPLC system consisted of an Agilent (Waldbronn, Germany) 1100 HPLC system (binary pump, degasser and autosampler) with diode array detector. LC separation was performed on a reversed phase (Phenomenex, Aschaffenburg, Germany $250 \times 4.6 \mathrm{~mm}$ i.d., $5 \mu \mathrm{m}$, Synergi polar RP), column thermostatted at $25^{\circ} \mathrm{C}$ using mobile phase $A$ (acetonitrile) and mobile phase B (water, acetic acid $0.5 \% \mathrm{v} / \mathrm{v}$ ) in a gradient program with a flow rate of $1 \mathrm{~mL} / \mathrm{min}: 0-9 \mathrm{~min}: 25 \%$ A; 9-20 $\mathrm{min}: 25-80 \%$ A; $20-$ 25 min: $80-25 \%$ A; 25-28 min: $25 \%$ A. Injection volume is $20 \mu \mathrm{L}$. For quantitative analysis, the wavelength with the highest intensity $(278 \mathrm{~nm})$ was used for both coumarin and safrole. Furthermore, UV/Vis spectra between 210 and $400 \mathrm{~nm}$ were recorded to verify the peak identity of coumarin and safrole.

\subsection{Sample Preparation}

\subsubsection{Barks/Powders}

For barks, an adequate amount of plant material was pulverized with a mortar and pestle. Dry plant samples $(0.5 \mathrm{~g})$ were weighed and sonicated in $20 \mathrm{~mL}$ of methanol for 30 min followed by centrifugation for 10 $\mathrm{min}$ at $3000 \mathrm{rpm}$. The supernatant was transferred to a $25-\mathrm{mL}$ volumetric flask. The procedure was repeated using $5 \mathrm{~mL}$ of methanol and respective supernatants were combined. The final volume was adjusted to 25 $\mathrm{mL}$ with methanol and mixed thoroughly. Prior to injection, an adequate volume (ca. $2 \mathrm{~mL}$ ) was passed through a $0.2-\mu \mathrm{m}$ nylon membrane filter. The first $1 \mathrm{~mL}$ was discarded and the remaining volume was collected in a LC sample vial.

\subsubsection{Rolls, Biscuits and Pastries}

For rolls, biscuits and pastries samples, a piece of bread roll was cut from each type. An adequate amount (50-100 g) was taken for sample homogenizing. The selected samples were cut into less than $0.5 \mathrm{~cm}$ cubes and mixed in a zip bag. Homogenized samples of bread or rolls $(3.0 \mathrm{~g})$ were weighed and sonicated in 20 $\mathrm{mL}$ of methanol for 30 min followed by centrifugation for $10 \mathrm{~min}$ at $3000 \mathrm{rpm}$. The supernatant was transferred to a $25-\mathrm{mL}$ volumetric flask. The procedure was repeated using $5 \mathrm{~mL}$ of methanol. The respective supernatants were combined, and the final volume was adjusted to $25 \mathrm{~mL}$ with methanol. The extracts were then processed as stated previously for barks.

\subsubsection{Tea Samples}

From tea leaves, a concoction was prepared as prescribed on the tea package. Depending on the prescription, $100-250 \mathrm{~mL}$ of $80-100^{\circ} \mathrm{C}$ hot water was added to $1.5-2 \mathrm{~g}$ of tea ingredients and incubated for 3$6 \mathrm{~min}$ prior to filtration and then processed as stated previously for barks.

\subsection{Preparation of Standard Solutions}

Individual stock solutions of the standard compounds were prepared at a concentration of 1 $\mathrm{mg} / \mathrm{mL}$ in methanol for coumarin and $1.6 \mathrm{mg} / \mathrm{mL}$ in methanol for safrole. Calibration curves were prepared using five different concentration levels.

\subsection{Validation Procedure}

The developed HPLC method was validated in terms of precision, accuracy, and linearity according to International Conference on Harmonisation of Technical Requirements for Registration of Pharmaceuticals for Human Use (ICH) guidelines [15]. The precision of the assay method was determined using three independent test solutions on three consecutive days. Recovery experiments were conducted using concentrations of the standards. The 
limit of detection (LOD) and limit of quantitation (LOQ) were estimated by injecting the dilute solutions of the standards with known concentration.

\subsection{Food Samples}

A total of 30 cinnamon and cinnamon-flavored food samples were collected as a part of an inspection program on the local market in Damascus, Syria. The program involved samples labeled with cinnamon from local bakery stores and supermarket storage facilities. Samples $(n)$ were included from the categories of cinnamon sticks and powder $(n=7)$, traditional and/or seasonal bakery ware $(n=6)$, fine bakery ware $(n=13)$ and tea $(n=4)$. The distinction between the categories of traditional and/or seasonal bakery ware and fine bakery ware is not specified in the current EU regulation [9]. At the DVFA (Danish Veterinary and Food Administration), bakery ware readily available to consumers all year round and not part of a traditional custom is placed in the category of fine bakery ware [16]. Bakery ware that is not normally consumed on a regular basis but consumed as a part of a tradition is placed in the category of traditional and/or seasonal bakery ware. The DVFA regard the high maximum limit (50 mg/kg) for traditional and/or seasonal bakery ware justified in the limited consumption of bakery ware in this category [16]. Upon arrival at the laboratory, samples were homogenized and frozen at $-4^{\circ} \mathrm{C}$ until further analysis.

\section{RESULTS AND DISCUSSIONS}

\subsection{HPLC Method Validation}

The HPLC method was validated for precision, accuracy, linearity, limits of detection, and limits of quantitation. Specificity of the method was established through determination of peak purity in samples with PDA detection at UV $278 \mathrm{~nm}$. The specificity of the HPLC method was determined by injecting individual standard samples. No interference was observed for any of the components. The purity of the principal and other chromatographic peaks was found to be satisfactory. The precision and stability of the assay method was evaluated by carrying out three independent assays on three different days. Multiple assays and injections showed that the results are highly reproducible and showed low standard error. The RSD of assay results obtained in interday and intraday study was within $2 \%$. The inter- and intraday assays confirmed the good precision of the method. The recoverability of the method was determined for the related substance by spiking samples with three different known amounts of each standard. The average recoveries of the analytes in sample S- 1 were $98.89 \%, 99.6 \%, 99.11 \%$ for coumarin standard and $98.5 \%, 99.12 \%, 98.7 \%$ for safrole standard. The LOD and LOQ were estimated by injecting the dilute solutions of the standards with known concentration. The LOQs were $1.9 \mu \mathrm{g} / \mathrm{ml}$ and $1.3 \mu \mathrm{g} / \mathrm{ml}$ for coumarin and safrole respectively. The LODs were $0.6 \mu \mathrm{g} / \mathrm{ml}$ and $0.4 \mu \mathrm{g} / \mathrm{ml}$ for coumarin and safrole respectively. LODs and LOQs were defined, respectively, as signal-tonoise ratio equal to 3 and 10 . Linear calibration plots for the related substances were obtained over the calibration range at five concentration levels. The linear dynamic range for coumarin $1.9-100 \mu \mathrm{g} / \mathrm{mL}$ and for safrole was $1.3-100 \mu \mathrm{g} / \mathrm{mL}$. The results showed good linear correlation $\left(r^{2}>0.999\right)$.

\subsection{Food Samples}

Coumarin and safrole were identified at retention times $10.4 \mathrm{~min}$. and $20 \mathrm{~min}$. respectively (Figure 1). In all samples, the UV profiles of coumarin and safrole were consistent with literature data [13] showing a global maximum at around $278 \mathrm{~nm}$. (Figures 2, 3).

A total of 30 cinnamon and cinnaomon-flavored food samples were analyzed in an inspection program on the local market in Damascus, Syria and compared to the EU limits for coumarin (European Parliament and

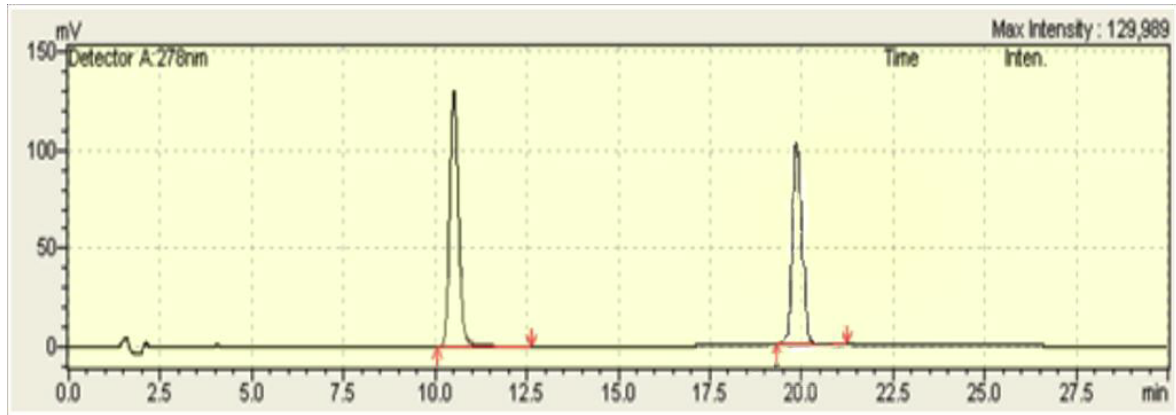

Figure 1: A chromatogram of coumarin and safrole standards at concentrations $0.1 \mathrm{mg} / \mathrm{mL}$ and $0.16 \mathrm{mg} / \mathrm{mL}$ respectively; obtained at a wavelength of $278.1 \mathrm{~nm}$. 


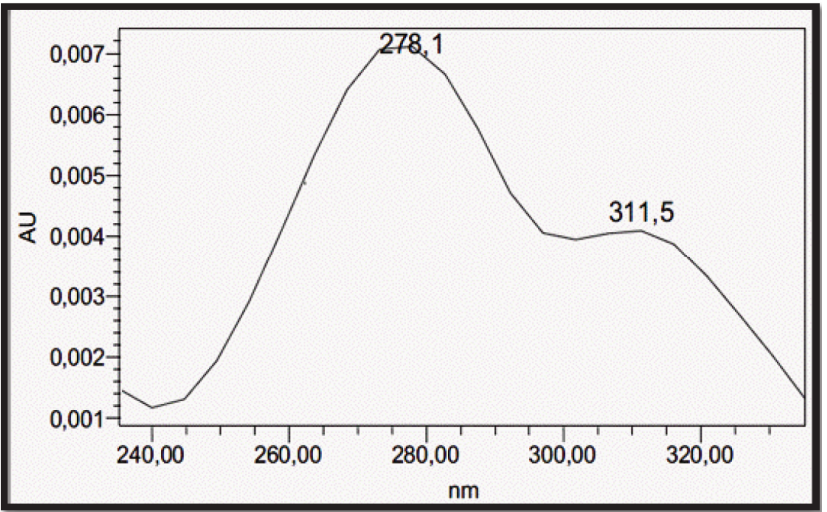

Figure 2: The UV profile $(240-340 \mathrm{~nm})$ obtained from coumarin eluted at $10.4 \mathrm{~min}$.

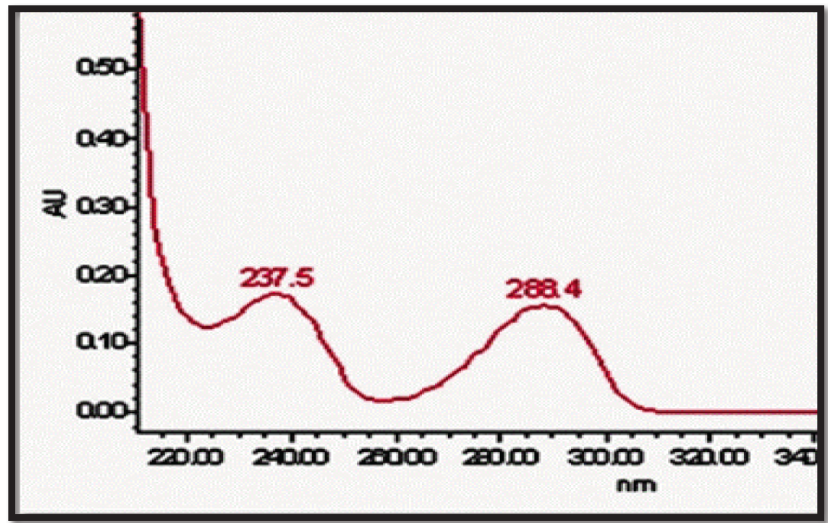

Figure 3: The UV profile $(220-340 \mathrm{~nm})$ obtained from safrole eluted at $20 \mathrm{~min}$.

Council, 2008). In the category of cinnamon sticks and powder 7 samples (S1-S7) were randomly collected from the market and analyzed. Analytical results for all cinnamon samples are reported in Figure 4. As expected, coumarin contents ranged from a few mg up to thousands $\mathrm{mg}$ per $\mathrm{kg}$ of cinnamon. Whereas no safrole levels were detected, indicating no contamination present in cinnamon powder by cinnamon leaf oil.
Regarding ground cinnamon, coumarin levels let suppose that the tested samples derive from cassia cinnamon [3]. The whole quill bark samples are also referable to Cassia cinnamon. Whole quill barks of cinnamon allow the identification of their origin. Cassia cinnamon is dark brown and rolled from both sides toward the centre so that they end up resembling scrolls. Moreover, cassia cinnamon is about $1.5 \mathrm{~mm}$ thick [3]. In the category of traditional and/or seasonal bakery ware, 6 samples (S8-S13) were analyzed and showed a content of coumarin ranging between 4.38 and $39.5 \mathrm{mg} / \mathrm{kg}$ with a mean coumarin content of 19.91 $\mathrm{mg} / \mathrm{kg}$ and, thus, not exceeding the EU limits for coumarin. In the category of fine bakery ware, 13 samples (S14-S16 and S21-S30) were analyzed and showed a content of coumarin ranging between 2 and $38.16 \mathrm{mg} / \mathrm{kg}$ with a mean coumarin content of 11.44 $\mathrm{mg} / \mathrm{kg}$. In this category, 5 samples exceeded the EU limits for coumarin. One sample contained $38.15 \mathrm{mg}$ coumarin $/ \mathrm{kg}$ and, thereby, exceeding the EU limit with more than a factor of two. No safrole contamination was detected in any of the samples (Figure 5).

Tea samples were also subjected to analysis despite the lack of EU limits for coumarin in these food products. Four samples of tea (ready to drink) with cinnamon were analyzed and showed a content of coumarin ranging from 0.119 to $0.28 \mathrm{mg} / \mathrm{tea}$ bag (one tea bag content is $1.5 \mathrm{~g}$ tea) with a mean coumarin content of $0.2 \mathrm{mg} / \mathrm{tea}$ bag (or $133 \mathrm{mg} / \mathrm{kg}$ ) (Figure 6). The normal daily intake of tea in Syria is large. In this context, not only the possible exceedance of the TDI may be relevant but also the higher bioavailability of coumarin in the case of a fluid matrix, especially, if the peak level is the critical parameter triggering the toxic effect (what is currently unknown) [17]. The limited number of samples disables a valid comparison of their coumarin content. It could be interesting to investigate

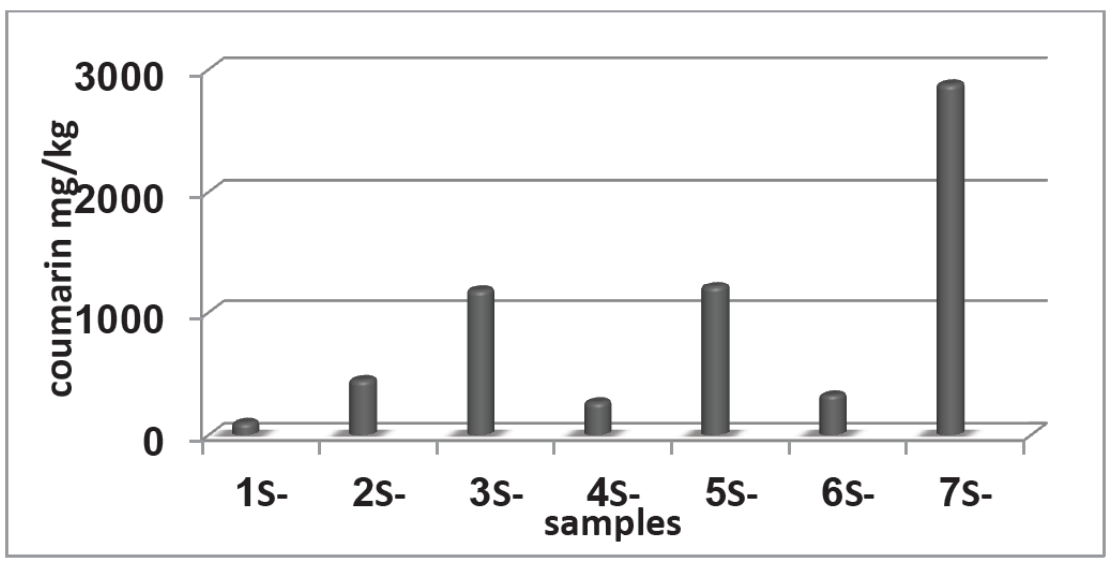

Figure 4: Analytical results for coumarin in cinnamon samples (mg/kg). 


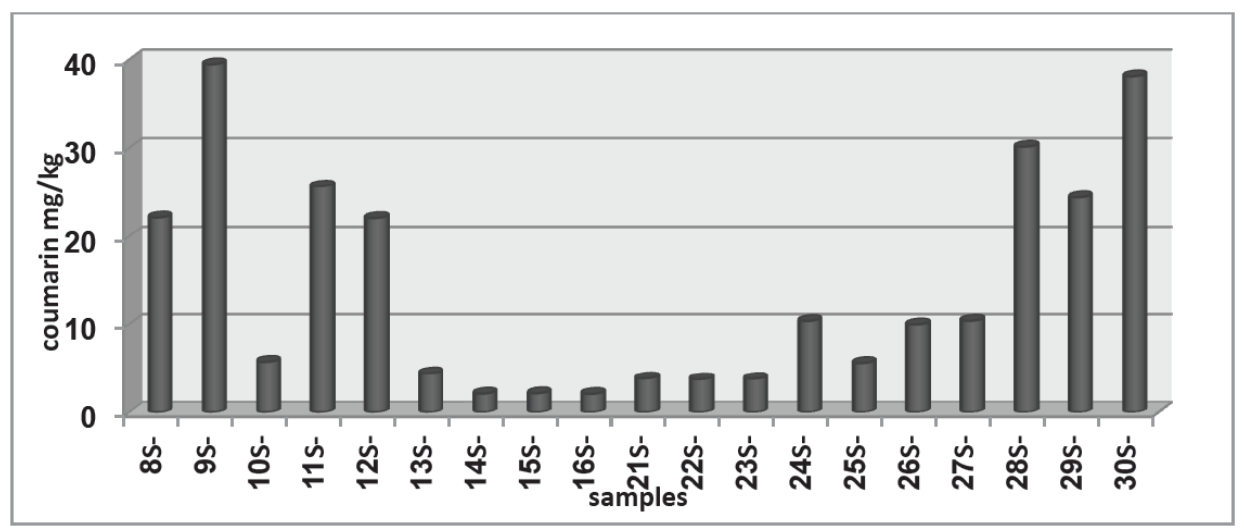

Figure 5: The content of coumarin in food products compared to the European Regulation (EC) No $1334 / 2008$.

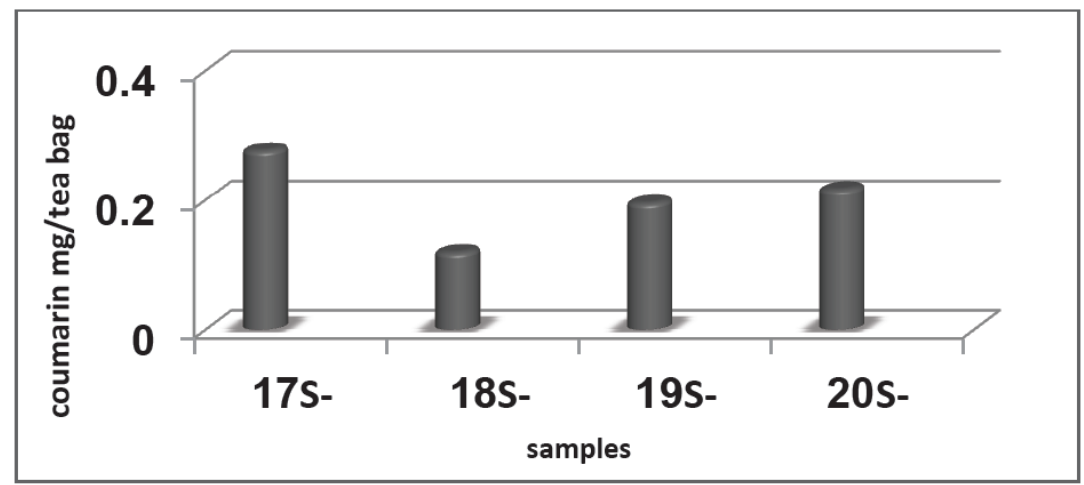

Figure 6: The content of coumarin (mg/tea bag) in Tea samples.

the coumarin content in a larger number of tea samples to evaluate the need for an EU limit for coumarin in tea.

\section{CONCLUSIONS}

The present HPLC method is rapid and suitable for routine testing of coumarin in food products with cinnamon. In this study, the presence of cinnamon in fine bakery ware has resulted in several cases of a coumarin content exceeding the EU limits. The reason for the high incidence of samples exceeding the EU limit for coumarin in bakery ware is unknown. However, a lack of knowledge about the EU regulation could be one explanation.

Tea is not included in the EU regulation. However, tea is an important consumable for many people and might contribute to the overall daily consumption of coumarin. In 2004, the European Food Safety Authority (EFSA) recommended a TDI of 0-0.1 $\mathrm{mg}$ coumarin $/ \mathrm{kg}$ body weight [6]. Children represent a sensitive group because of a low body weight and a dietary habit towards sweet food with cinnamon. A child of $30 \mathrm{~kg}$ that consumes $250 \mathrm{ml}$ of tea with a content of $12 \mathrm{mg}$ coumarin/l has reached this TDI. In this case, all the TDI of coumarin is allocated to tea, which could indicate that tea might have a problematic high content of coumarin. It could, therefore, be interesting to gather more data on the coumarin content in tea, to consider its inclusion in a future EU regulation.

\section{REFERENCES}

[1] Clark GS. Coumarin: An aroma chemical profile. Perfumer \& Flavorist 1995; 20: 3-34.

[2] Lake BG. Coumarin metabolism, toxicity and carcinogenicity: relevance for human risk assessment. Food Chem Toxicol 1999; 37: 423-453. https://doi.org/10.1016/S0278-6915(99)00010-1

[3] Ravindran PN, Nirmal-Babu K, Shylaja M. Introduction in Cinnamon and Cassia: The genus Cinnamomum. Boca Raton: CRC Press 2004; pp. 1-13.

[4] Blahova J, Svobodova Z. Assessment of coumarin levels in ground cinnamon available in the Czech retail market. Scientific World J 2012; 26: 38-51. https://doi.org/10.1100/2012/263851

[5] Miller KG, Poole CF, Pawloski TM. Classification of the botanical origin of cinnamon by solid-phase microextraction and gas chromatography. J Chromatogr 1996; 42: 639-46. https://doi.org/10.1007/BF02267695

[6] EFSA. Opinion of the scientific panel on food additives, flavourings, processing aids and materials in contact with food (AFC) related to Coumarin. EFSA J 2004; 104: 1-36.

[7] Abraham K, Pfister M, Wöhrlin F, et al. Relative bioavailability of coumarin from cinnamon and cinnamon-containing foods compared to isolated coumarin: a four-way crossover study in human volunteers. Mol Nutr Food Res 2011; 55: 644-53. https://doi.org/10.1002/mnfr.201000394 
[8] European Council. Council Directive of 22 June 1988 on the approximation of the laws of the member states relating to flavourings for use in foodstuffs and to source materials for their production. OJEU 88/388/EEC 1988; p. 1-10.

[9] European Parliament and Council. Regulation (EC) no $1334 / 2008$ of the European Parliament and of the council of 16 December 2008 on flavourings and certain food ingredients with flavouring properties for use in and on foods and amending council regulation (EEC) no 1601/91, regulations (EC) no 2232/96 and (EC) no 110/2008 and directive 2000/13/EC. OJEU 2008; 354: 34-50.

[10] Carlson M, Thompson RD. Liquid chromatographic determination of safrole in sassafras-derived herbal products. J AOAC Int 1997; 80: 1023-28.

[11] Bisset N. Sassafras lignum. in Herbal Drugs and Phytopharmaceuticals, Stuttgart, Germany: CRC Press 1994; pp. 455-56.

[12] EEC. Council Directive 88/388/EEC of 21 June 1988 on the approximation of the laws of the member States relating to flavourings for use in foodstuffs and to source materials for their production. OJEU 1988; 184: 61-67.
[13] Maggi F, Barboni L, Caprioli G, et al. HPLC quantification of coumarin in bastard balm (Melittis melissophyllum L., Lamiaceae). Fitoterapia 2011; 82: 1215-21. https://doi.org/10.1016/j.fitote.2011.08.011

[14] Choong YM, Lin HJ. A Rapid and Simple Gas Chromatographic Method for Direct Determination of Safrole in Soft Drinks. J Food Drug Anal 2001; 9: 27-32.

[15] ICH Harmonised Tripartite Guideline. Validation of analytical procedures: text and methodology Q2 (R1) 2005; http://www.ich.org/fileadmin/Public_Web_Site/ICH_Products/ Guidelines/Quality/Q2_R1/Step4/Q2 R1 Guideline.pdf, (Accessed April 8, 2013).

[16] Ballin NZ, Sørensen AT. Coumarin content in cinnamon containing food products on the Danish market. Food Control 2014; 38: 198-203. https://doi.org/10.1016/j.foodcont.2013.10.014

[17] Abraham K, Wöhrlin $\mathrm{F}$, Lindtner $\mathrm{O}$, et al. Toxicology and risk assessment of coumarin: focus on human data. Mol Nutr Food Res 2010; 54: 228-39. https://doi.org/10.1002/mnfr.200900281 